\title{
Laparoscopic Management of Large Ovarian Cysts
}

\author{
${ }^{1} \mathrm{CS}$ Beeresh, ${ }^{2}$ Divyasree Doopadapalli, ${ }^{3} \mathrm{KR}$ Vimala, ${ }^{4} \mathrm{Krishna}$ Lingegowda
}

\begin{abstract}
Introduction: Large ovarian cysts are conventionally managed by laparotomy. This study was undertaken to assess the feasibility and outcome of laparoscopic surgery for the management of large ovarian cysts.
\end{abstract}

Settings and design: Rural teaching hospital - prospective study.

Materials and methods: Thirty-eight patients from January 2014 to December 2016 presumed to be with large ovarian cyst were managed laparoscopically. Preliminary evaluation suggestive to be of benign ovarian cyst by history, clinical examination, sonographic imaging, and basic serum marker were only included in this study. The cysts were aspirated initially, followed by cystectomy, oophorectomy, or total hysterectomy depending on age, parity, coexisting pathology, and desire for future fertility.

Results: Out of 38 cases, 6 were nonovarian adnexal masses. Eight of the 32 cases who presented with pain due to torsion were managed on emergency basis; rest of the cases were operated electively. Mean operating time was 90 minutes. Mean size of the cyst was $16 \mathrm{~cm}$. One case of borderline malignancy was detected and the rest showed benign pathology. Six of the cases required minilaparotomy for specimen removal. Most women were successfully treated laparoscopically without any complications, and conversion to laparotomy was required in three cases.

Conclusion: With proper patient selection and exclusion of malignancy, laparoscopic management of large ovarian cyst by gynecologist is feasible.

Keywords: Benign ovarian cyst, Laparoscopy, Large ovarian cysts.

How to cite this article: Beeresh CS, Doopadapalli D, Vimala KR, Lingegowda K. Laparoscopic Management of Large Ovarian Cysts. Int J Gynecol Endsc 2017;1(1):18-21.

Source of support: Nil

Conflict of interest: None

\section{INTRODUCTION}

Surgical treatment has become more conservative and less invasive. Thus, operative laparoscopy is now considered as the gold standard for surgical management of gynecological condition. The advantages of laparoscopic surgery

\footnotetext{
${ }^{1,2}$ Assistant Professor, ${ }^{3}$ Professor, ${ }^{4}$ Professor and Head

${ }^{1-4}$ Department of Obstetrics and Gynecology, PES Institute of Medical Sciences \& Research, Chittoor, Andhra Pradesh, India

Corresponding Author: CS Beeresh, No 101, E block, Staff quarters, PES Medical College, Chittoor, Andhra Pradesh, India Phone: +91-9449316479, e-mail: beeresh.25@gmail.com
}

include small incisions, less postoperative pain, short hospital stay, early ambulation, early return to normal activities, decreased chance of deep venous thrombosis and pulmonary embolism, fewer complications related to the incision, and better patients' satisfaction and quality of life. Despite being performed through small incisions, the visualization of the operative field, surgical outcome, and the ability to achieve the surgical objectives have been similar among patients undergoing laparoscopic surgery compared with those whose surgery was performed through laparotomy.

Ovarian neoplasm is a common clinical entity affecting women of all age groups. Up to $10 \%$ of women will have some form of surgery during their lifetime for the presence of an ovarian mass. ${ }^{1}$ Most of these ovarian neoplasms are benign, and the overall incidence of asymptomatic ovarian cyst in a premenopausal female being malignant is only approximately 1:1,000, increasing to 3:1,000 at the age of 50 years. So, most of these benign neoplasms can be managed by a gynecologist at their peripheral centers.

The definition of large ovarian cyst is not clearly described in the literature. Some authors describe large ovarian cyst as those measuring above $10 \mathrm{~cm}$ sonologically, $^{2}$ whereas others describe clinically as those reaching above umbilicus. ${ }^{3}$ Although a maximum tumor size above which minimal access surgery is contraindicated has not been determined, some have suggested that ovarian masses that are $10 \mathrm{~cm}$ in size are best managed by laparotomy because of anticipated technical difficulty with the procedure as well as the perceived higher malignant potential of large adnexal masses.

We report a 2-year experience with the laparoscopic management of adnexal masses more than $10 \mathrm{~cm}$ in size to determine the feasibility.

\section{MATERIALS AND METHODS}

All women presenting with adnexal mass more than $10 \mathrm{~cm}$ during January 2014 to December 2016 (2 years) to our rural teaching institution were considered for this study. Preoperative evaluation included history, clinical examination, ultrasound examination, and basic serum marker evaluation (CA-125 upper limit 35 U/mL). Inclusion criteria included ovarian cyst of more than $10 \mathrm{~cm}$ with low probability of malignancy. Cases with ascites, solid/complex mass, enlarged lymph nodes, and elevated CA-125 were excluded from study. 
Written informed consent was obtained from the patients after a thorough counseling, detailing therapeutic options, risks of the procedure, and the need for possible laparotomy or other indicated procedures. All procedures were performed by surgeons with training and experience in laparoscopic procedures.

In all cases, $10 \mathrm{~mm}$ primary port was accessed supraumbilically either by open laparoscopy or by creating pneumoperitonium depending on the case. Three $5 \mathrm{~mm}$ working ports were created, one on each side an inch above and medial to superior iliac spine, and a third port on the left side midway between the first port and telescope. The cyst wall, interior of the capsule, and the visceral organs were examined systematically for any suspicious signs of malignancy.

The cysts were aspirated initially followed by cystectomy, oophorectomy, or total hysterectomy depending on age, parity, coexisting pathology, and desire for future fertility. In cystectomy, capsule stripped from remaining ovarian tissue using graspers and bipolar current was used to coagulate the bleeding surface. If cystectomy was not performed or was not feasible, then cystic ovary (along with fallopian tube in some cases) was mobilized and separated from its attachments using bipolar coagulation and cold scissors. If associated with other uterine pathologies, salpingo-oophorectomy followed by hysterectomy was performed and the entire specimen was delivered vaginally. Specimen was retrieved by any of the three methods: (1) En masse along with cannula - through $10 \mathrm{~mm}$ primary port held with grasper, (2) in bits and pieces - through $10 \mathrm{~mm}$ port after cutting into small strips, or through colpotomy. We resorted to minilaparotomy, if all the above methods failed.

After the tissue was removed, the abdominal and pelvic cavities were thoroughly irrigated with copious

Table 1: Symptoms of patients with ovarian cyst

\begin{tabular}{ll}
\hline Symptoms & Patients $(n)$ \\
\hline Acute pain abdomen & 8 \\
Infertility & 5 \\
Abdominal distension and discomfort & 16 \\
Incidental finding & 3 \\
\hline Total & 32 \\
\hline
\end{tabular}

Table 3: Specimen retrieval

\begin{tabular}{ll}
\hline Specimen retrieval method & Number $(n)$ \\
\hline Vagina (after hysterectomy) & 10 \\
Colpotomy - through posterior fornix & 7 \\
Through port incision & 6 \\
Minilaparotomy & 6 \\
Laparotomy (conversion) & 3 \\
\hline Total & 32 \\
\hline
\end{tabular}

amounts of normal saline. All the patients were discharged on 3rd or 4th postoperative day with standards of care at our institution.

\section{RESULTS}

The mean age of the patients was 37 years (ranging from 22 to 69 years). Six patients were postmenopausal. Eight patients presented with features of torsion (Table 1).

Most of the cases could be completed laparoscopically without any complication, and conversion to laparotomy was needed in three cases $(9.3 \%)$ to achieve desired surgical outcome (two cases of severe endometriosis, one to arrest bleeding). The average diameter of the cyst was $16 \mathrm{~cm}$ $(10-28 \mathrm{~cm})$. Patients underwent cystectomy, adnexectomy, or total hysterectomy depending on age, parity, desire for future fertility, and operative feasibility. Ten patients underwent laparoscopic adnexectomy followed by hysterectomy (prolapse two, cervical dysplasia two, fibroids two, dysfunctional uterine bleeding four) (Table 2).

Specimen retrieval - the specimen was delivered through vagina in all patients undergoing hysterectomy following adnexectomy $(n=10)$. Suprapubic minilaparotomy incision of $3 \mathrm{~cm}$ was required to deliver the necrotic tissue (due to torsion) in six cases, as the specimen had hardened secondary to stagnation of blood (Table 3).

Mean duration of surgery was 90 minutes (70-135 minutes). Patients had uneventful postoperative period and was discharged on 3rd or 4th postoperative day. All cases had benign pathology and the most common was serous cyst adenoma (Table 4).

\section{DISCUSSION}

A randomized prospective study comparing laparoscopy and laparotomy in the management of patients

Table 2: Laparoscopic procedure

\begin{tabular}{ll}
\hline Procedure & $\begin{array}{l}\text { Patients } \\
(n=32)\end{array}$ \\
\hline Laparoscopic cystectomy & 6 \\
Laparoscopic oophorectomy/salpingo-oophorectomy & 13 \\
Laparoscopic adnexectomy followed by hysterectomy & 10 \\
Laparoscopy followed by laparotomy (conversion) & 3 \\
\hline Total & 32 \\
\hline
\end{tabular}

Table 4: Histopathology of specimen

\begin{tabular}{ll}
\hline Histopathology & Patients $(n)$ \\
\hline Serous cyst adenoma & 14 \\
Mucinous cystadenoma & 9 \\
Dermoid & 4 \\
Endometrioma & 4 \\
Borderline serous cystadenoma & 1 \\
\hline Total & 32 \\
\hline
\end{tabular}




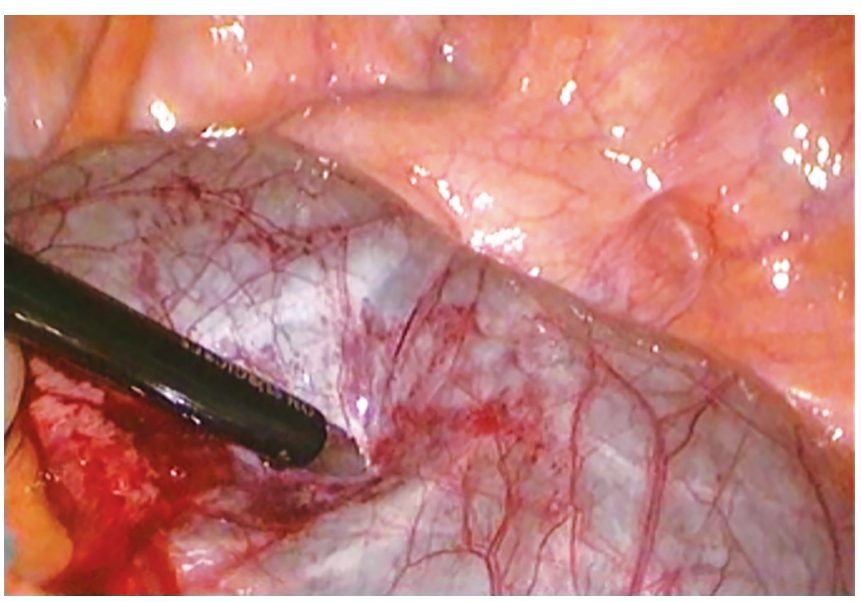

Fig. 1: Intentional trocar puncture of the cyst under visualization to deflate the cyst

with benign ovarian masses less than $10 \mathrm{~cm}$ in diameter reported a significant reduction in operative morbidity, postoperative pain and analgesic requirement, hospital stay, and recovery period. ${ }^{4}$

However, the laparoscopic approach for the removal of cysts greater than $10 \mathrm{~cm}$ presents certain difficulties.

First, large ovarian cysts occupy major portion of abdominal cavity causing difficulty in peritoneal access, visualization of vital structures, and restriction of working space. Various techniques have been described in the literature to overcome this difficulty, such as (1) Palmer's point access ${ }^{5}$ or (2) open laparoscopy ${ }^{6}$ followed by intentional trocar puncture of the cyst under visualization to deflate the cyst (Figs 1 and 2). In this study, both the above techniques were adopted depending on the case and without much difficulty. In one study, transabdominal drainage under ultrasonographic control followed by laparoscopy ${ }^{7}$ and port/Veress needle insertion directly into the cyst blindly has also been described. ${ }^{8}$ Since these two approaches later appear to be more blind technique compromising the safety of patient, these were not attempted in our cases.

Second, the risk of spillage of the cyst contents is associated with complications, such as pseudomyxoma peritonei (mucinous cystadenoma), chemical peritonitis (dermoid cyst), and the potential dissemination of unsuspected malignancy. Spillage rate depends on the cyst size, surgical expertise, and route of retrieval. Various techniques described to minimize spilling are: (1) closure of the cyst puncture site with a $5 \mathrm{~mm}$ grasper after deflating it and the adnexal extracted onto the abdomen wall through a minilaparotomy followed by extracorporeal cystectomy; ${ }^{9}$ (2) placement of purse suture or endoscopic loop around the cyst incision; and (3) specimen retrieval bags. Although described, these methods do not completely prevent spillage and is associated with increased operative time, hernia chances, operative cost, and hence,

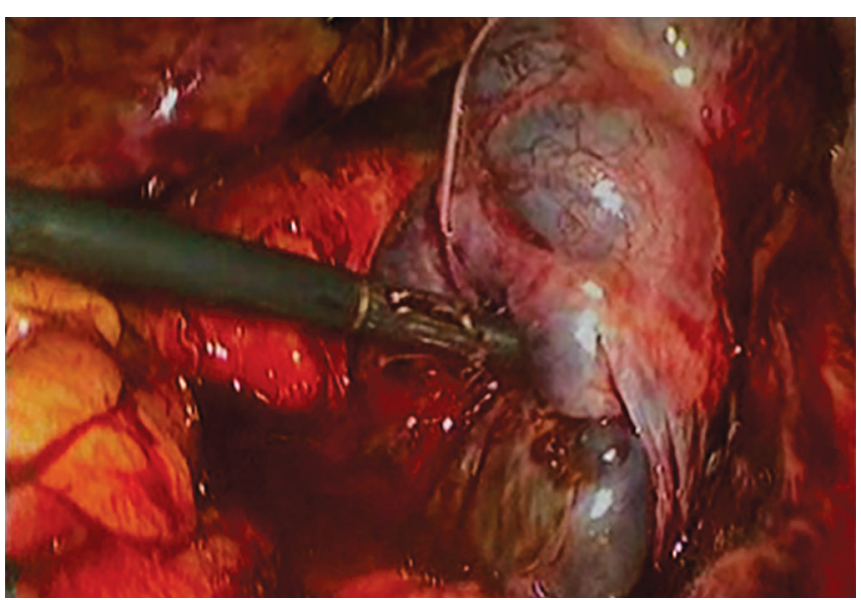

Fig. 2: Large endometriotic cyst

Table 5: Comparison of results

\begin{tabular}{lll}
\hline Results & $\begin{array}{l}\text { Review article } \\
(n=852)(\%)\end{array}$ & $\begin{array}{l}\text { Present study } \\
(n=32)(\%)\end{array}$ \\
\hline $\begin{array}{l}\text { Conversion to laparotomy } \\
\text { Intra- and postoperative }\end{array}$ & 9.3 & 3.9 \\
$\begin{array}{l}\text { complication } \\
\text { Borderline tumor }\end{array}$ & 1.9 & 0 \\
Malignancy & 2.5 & 3.1 \\
\hline
\end{tabular}

were not adopted in our study. In our study, we had nine cases of mucinous cystadenoma and four dermoid and one borderline serous cystadenoma and none had spillrelated complications.

Third was the longer operative time and technical difficulty associated with retrieval of larger tissue masses. Minilaparotomy ${ }^{10}$ is considered an acceptable minimally invasive approach yielding similar results. In our study, minilaparotomy was resorted to in six cases for specimen retrieval. Posterior colpotomy ${ }^{11}$ was successfully used in seven cases to deliver specimen out. In six cases, $10 \mathrm{~mm}$ port incision was used for specimen retrieval either en masse or after cutting into smaller bits. In another 10 cases, specimen was delivered through vaginal vault after hysterectomy.

Every attempt was made to conserve the ovarian tissue in all women of reproductive age. However, owing to the large size of the cyst, such an attempt was not possible, either because it was technically difficult or there was no ovarian tissue left in such huge cyst. Out of 24 reproductive age group patients, only in $8(33 \%)$ cystectomy could be performed in our study.

The results of the review article comparing 20 studies, including 852 patients of laparoscopic management of large or huge ovarian cysts, were comparable to our study (Table 5). ${ }^{12}$

Both in review and in our study, the most common was serous or mucinous cystadenoma. 


\section{CONCLUSION}

With proper patient selection, the size of an ovarian cyst should not constitute a contraindication to laparoscopic surgery.

\section{REFERENCES}

1. Royal College of Obstetricians and Gynaecologists. Management of suspected ovarian masses in premenopausal women. Green-top guideline No. 62 RCOG/BSGE Joint guideline I. 2011 Nov [cited 2017 Jan 25]. Available from: http://www. rcog.org.uk/guidelines.

2. Paul PG, Chopade G, PatilS, Das T, Thomas M, Garg R. Should we manage large ovarian cysts laparoscopically? J Gynecol Surg 2016 Oct;32(5):251-256.

3. Salem HA. Laparoscopic excision of large ovarian cysts. J Obstet Gynaecol Res 2002 Dec;28(6):290-294.

4. Yuen PM, Yu KM, Yip SK, Lau WC, Rogers MS, Chang A. A randomized prospective study of laparoscopy and laparotomy in the management of benign ovarian masses. Am J Obstet Gynecol 1997 Jul;177(1):109-114.
5. Stitely ML. Laparoscopic removal of a large ovarian mass utilizing planned trocar puncture. JSLS 2012 Jan-Mar;16(1):148-150.

6. Shindholimath VV, Jyoti SG, Patil KV, Ammanagi AS. Laparoscopic management of large ovarian cysts at a rural hospital. J Gynecol Endosc Surg 2009 Jul;1(2):94-97.

7. Nagele F, Magos AL. Combined ultrasonographically guided drainage and laparoscopic excision of a large ovarian cyst. Am J Obstet Gynecol 1996 Nov;175(5):1377-1378.

8. Quinlan DK. The laparoscopic management of large ovarian cysts. J Obstet Gynecol India 2010 Mar-Apr;60(1):152-156.

9. Göçmen A, Atak T, Uçar M, Sanlikal F. Laparoscopy-assisted cystectomy for large adnexal cysts. Arch Gynecol Obstet 2009 Jan;279(1):17-22.

10. Panici PB, Palaia I, Bellati F, Pernice M, Angioli R, Muzii L. Laparoscopy compared with laparoscopically guided minilaparotomy for large adnexal masses: a randomized controlled trial. Obstet Gynecol 2007 Aug;110(2 Pt 1):241-248.

11. Teng FY, Muzsnai D, Perez R, Mazdisnian F, Ross A, Sayre JW. A comparative study of laparoscopy and colpotomy for the removal of ovarian dermoid cysts. Obstet Gynecol 1996 Jun;87(6):1009-1013.

12. Eltabbakh G. Laparoscopic surgery for large ovarian cystsreview. Curr Trends Gynecol Oncol 2016;1:3. 